\title{
A Novel Color Encoding Fringe Projection Profilometry based on Wavelet Ridge Technology and Phase-Crossing
}

\author{
Yang Wang ${ }^{\mathrm{a}, \mathrm{b},{ }^{*}}$, Yankee Sun ${ }^{\mathrm{b}}$, Tianqi Zhang ${ }^{\mathrm{b}}$, Deyun $\mathrm{Chen}^{\mathrm{a}}$, and Xiaoyang $\mathrm{Yu}^{\mathrm{a}}$ \\ ${ }^{a}$ Postdoctoral Station of Computer Science and Technology, Harbin University of Science and Technology, Harbin, 150080, China \\ ${ }^{b}$ Higher Educational Key Laboratory for Measuring \& Control Technology and Instrumentations of Heilongiang Province, Harbin University of \\ Science and Technology, Harbin, 150080, China
}

\begin{abstract}
There are two challenges in three-dimensional (3D) profilometry, such as real-time and accuracy. Color-encoding fringe projection profilometry (CEFPP) can solve these challenges to some extent. It encodes in the red, green, and blue color channels, and CEFP can use three completely different fringe patterns. In this paper, a novel CEFP that only uses one color fringe image acquired by experiential 3CCD cameras is presented. First, the wavelet transform coefficients phase at the ridge position under the Morlet wavelet is theoretically clarified. A simple and quick method that acquires the scaling coefficient is introduced. The wrapped phases in the three color channels of the color fringe image are obtained by wavelet bridge position. The phase original of color fringe pattern is defined as one white color. Using an evolution function defined in this paper, the phase-crossing is located. An absolute phase is acquired by a three coding pitch method in a two coding direction. In order to verify the presented method, some profilometry experiments are carried out in the constructed 3D profilometry system using one projector and one 3CCD camera. The experimental results showed that a maximum standard deviation of measurement error is $1.42 \mathrm{~mm}$, and the reconstruction surface of a gypsum head portrait can be determined.
\end{abstract}

Keywords: color-encoding fringe projection profilometry; color image channel; wavelet ridge technology; phase-crossing

(Submitted on February 16, 2018; Revised on March 21, 2018; Accepted on April 28, 2018)

(C) 2018 Totem Publisher, Inc. All rights reserved.

\section{Introduction}

There is a demand of no-contact measurement of an object in many areas, such as biomedicine, surface topography, machine vision, industry inspection, reverse engineering and other applications [1,2]. Optical measurement methods meet this demand and are the effective method. Optical non-contact measurement has played an increasingly important role in these areas. Numerous techniques have been developed, including Moiré, holography, and fringe projection, which has become the first choice.

Fringe projection profilometry (FPP) based on fringe coding patterns and fringe image calculation has been employed. The coding-objects of fringe patterns are the pixel coordinates in the fringe patterns and are indicated as a cosine function $[3,4,5]$. They can be divided into Gray-encoded fringe projection profilometry [6,7] and Color-encoded fringe projection profilometry (CEFPP) $[8,9,10]$, etc.

There are two challenges in three-dimensional (3D) profilometry, such as real-time and accuracy. The color-encoding fringe projection profilometry (CEFPP) can solve these challenges to some extent [11,12]. CEFPP uses only one fringe pattern [13], which has three different fringe patterns encoded in the red (R), green (G), and blue (B) channels [14]. The measured object's surface is projected by this color fringe pattern. 3 CCD cameras are used to capture the corresponding color deformed fringe image. The diversity phase is extracted from three color channels of the deformed fringe image [15].

The phase information was extracted from fringe images by the acquire phase. According to the mathematical formula 
of the acquired phase, these methods can be divided into Phase shifting profilometry (PSP) $[16,17,18]$ and Fourier transformation profilometry (FTP) $[19,20]$. But, the spatial localization is poor in the FTP operation. The Windowed Fourier transform profilometry (WFTP) [21] has improved the spatial localization to some extent. Wavelets transform (WT) $[22,23,24]$ has advantages of multi-scaling frequency and locating temporal-spatial position. Because of these two advantages, WT has been used to analyze optical fringe images [25,26]. A wavelet ridge technique (WRT) is a technique used to locate the corresponding time or frequency of the single. Chen used WRT [27] to detect the spatial frequency of a deformation fringe image. After WT of the optical fringe image, the phase gradient method was introduced to obtain the phase via Watkins [28,29]. The Paul wavelet was then used to evaluate the phase distribution of fringe images [30,31]. All above methods focused on the wavenumber of fringe images as well as the scaling coefficient.

The result of the arctangent operation is a value in $(-\pi, \pi)$. WRT would obviously result in the phase of every color channel within $2 \pi$ discontinuities. In order to remove the $2 \pi$ discontinuities, many unwrapping phase methods have been proposed by scholars. The spatial unwrapping methods [32,33,34], such as the wrapped phase region growing method, the unwrapping path optimum method, the unwrapping phase quality navigation method and so on, are carried out by using one wrapped map. The absolute phase direct acquirement methods are another kind of method. These methods use not only the wrapped phase map, but also other symbols [35,36,37,38]. These symbols include another wrapped phase map (dualwavelength method) and the coding pitch symbols (Gray-coding or other binary code). However, all these methods started to form the end of the fringe image. This beginning wastes another coding direction of fringe patterns.

According to the definition of a Morlet wavelet, the wavelet bridge position is theoretically demonstrated. Then, we clarified that the relationship between the scaling coefficient and the square root of a wavenumber fringe image deformed from the fringe pattern is inversely proportional [39,40,41]. These are mainly explained in Section 2. Section 3 introduces a simple method of selecting the scaling the coefficient vector $\mathrm{p}$. The color coding fringe pattern and a novel absolute phase acquirement is proposed. The color coding fringe pattern and a novel absolute phase acquirement method is given in Section 4. Section 5 shows the experimental results. Finally, conclusions are made in Section 6.

\section{Phase Acquirement Method by WRT}

\subsection{Wavelet Ridges}

A mother wavelet could convert to serious daughter wavelets by changing parameters. Firstly, daughter wavelets $\varphi p, t(x)$ are normalized by the inverse square root factor such as

$$
\varphi_{p, t}(x)=\frac{1}{\sqrt{p}} \varphi\left(\frac{x-t}{p}\right)
$$

where $t$ is a shift parameter related to $x$ and $p$ is a scaling coefficient and $p>0$. This normalization of a daughter wavelet helps the scaling coefficient $p$ dissociate from the single energy or the amplitude of the WT coefficients. By using this normalization, all frequency components are equal. With this definition, the WT coefficient, $\mathrm{W}(\mathrm{p}$, $\mathrm{t})$, of one fringe image can be obtained by

$$
W(p, t)=\left\langle I(x), \varphi_{p, t}(x)\right\rangle=\int_{-\infty}^{\infty} I(x) \varphi_{p, t}^{*}(x) d x
$$

where $\varphi^{*}$ is the complex conjugate function of the mother wavelet $\varphi$.

Because the complex Morlet has a spatial localization, this is a selection mother wavelet. Its form is described as

$$
\varphi(x)=\frac{1}{\sqrt{\pi F_{B}}} \exp \left(j 2 \pi F_{C} x\right) \exp \left(-\frac{1}{F_{B}} x^{2}\right)
$$

where FB is the wavenumber range concluded in the mother wavelet, and FC represents its middle wavenumber of FB. The gray value of an optical fringe image can be expressed as

$$
I(x)=A+B \cos \phi(x)=A+B \frac{e^{i \phi(x)}+e^{-i \phi(x)}}{2}
$$

where (x) is the phase distribution of the fringe image modulated by the measured object. A represents the ambient light, usually as DC components. B denotes the amplitude modulation of the fringes, and $\mathrm{i}$ is the imaginary unit. According to formula (2), The WT of I(x) is as follows. 


$$
\begin{aligned}
W(p, t) & =\int_{-\infty}^{\infty} I(x) \varphi_{p, t}^{*}(x) d x=\int_{-\infty}^{\infty} A \varphi_{p, t}^{*}(x) d x+\int_{-\infty}^{\infty} B e^{i \phi(x)} \varphi_{p, t}^{*}(x) d x+\int_{-\infty}^{\infty} B e^{-i \phi(x)} \varphi_{p, t}^{*}(x) d x \\
& =W_{1}+W_{2}+W_{3}
\end{aligned}
$$

Employing the complex Morlet definition in Formula (3), the WT in Formula (5) becomes

$$
\begin{aligned}
& W_{1}=\int_{-\infty}^{\infty} A \varphi_{p, t}^{*}(x) d x \\
& W_{2}=\frac{1}{2} \sqrt{p} B \exp \left\{-\left\{\pi F_{C} \sqrt{F_{B}}\left[\frac{\phi^{\prime}(t) p}{2 \pi F_{C}}-1\right]\right\}^{2}\right\} \exp [i \phi(t)] \\
& W_{3}=\frac{1}{2} \sqrt{p} B \exp \left\{-\left\{\pi F_{C} \sqrt{F_{B}}\left[\frac{\phi^{\prime}(t) p}{2 \pi F_{C}}+1\right]\right\}^{2}\right\} \exp [-i \phi(t)]
\end{aligned}
$$

Usually, the wavenumber of one fringe image is more than 1, so the coefficient $\mathrm{FB}>1$. FC $>1$ is obviously satisfies. If (b) $>0$, then $\mathrm{W} 3=0$. This case is the same when (b) $<0$ and W2 $=0$. The WT coefficient of $\mathrm{I}(\mathrm{x})$ can be calculated as $\mathrm{W}=\mathrm{W}(\mathrm{p}$, $\mathrm{t})=\mathrm{W} 2($ or $\mathrm{W}=\mathrm{W}(\mathrm{p}, \mathrm{t})=\mathrm{W} 3)$. The modulus of the WT coefficient $\mathrm{I}(\mathrm{x})$ is expressed as follows.

$$
|W|=\left|\frac{1}{2} \sqrt{p} B \exp \left\{-\left\{\pi F_{C} \sqrt{F_{B}}\left[\frac{\phi^{\prime}(t) p}{2 \pi F_{C}}-1\right]\right\}^{2}\right\} \exp [i \phi(t)]\right|
$$

Thus, the partial derivatives of the modulus of the WT coefficient $|\mathrm{W}|$ to the scaling coefficient $\mathrm{p}$ can be calculated as

$$
\frac{\partial|W|}{\partial p}=\sqrt{p} B \exp \left\{-\left\{\pi F_{C} \sqrt{F_{B}}\left[\frac{\phi^{\prime}(t) p}{2 \pi F_{C}}-1\right]\right\}^{2}\right\} \times\left\{\frac{1}{4}-\left\{\pi F_{C} F_{B}\left[\frac{\phi^{\prime}(t) p}{2 \pi F_{C}}-1\right]\right\} \frac{\phi^{\prime}(t) p}{2}\right\}
$$

Setting this partial derivative as zero, $|\mathrm{W}|$ reaches its maximum value at

$$
p_{r}(t)=\frac{\pi F_{B} F_{C}+\sqrt{\left(\pi F_{B} F_{C}\right)^{2}+F_{B}}}{F_{B} \phi^{\prime}(t)}=\frac{\pi F_{B} F_{C}+\sqrt{\left(\pi F_{B} F_{C}\right)^{2}+F_{B}}}{2 \pi F_{B}} \frac{1}{f_{B}}
$$

where $\operatorname{pr}(\mathrm{t})$ represents the scaling coefficient at the ridge point dependent on the spatial shift parameters $\mathrm{t}$, and $\mathrm{fB}=$ (t) $/ 2 \pi$ is the so-called wavenumber of the fringe image. Position $t$, the wavelet ridge of the WT coefficient is defined as the modulus peak of the WT coefficient. Therefore, the WT coefficient of the optical fringe image on the wavelet ridge becomes

$$
W_{M}\left(p_{r}, t\right)=\frac{1}{2} B \sqrt{p_{r}(t)} \exp \left\{-\left\{\pi F_{C} \sqrt{F_{B}}\left[\frac{\pi F_{B} F_{C}+\sqrt{\left(\pi F_{B} F_{C}\right)^{2}+F_{B}}}{2 \pi F_{B} F_{C}}-1\right]\right\}^{2}\right\} \exp [i \phi(t)]
$$

At the wavelet ridge position, the modulus of the WT coefficients has a positive relationship with the amplitude modulation of the fringes as well as the square root of the scaling coefficient $\mathrm{p}$. This modulus has an inverse relationship with the square root of the wavenumber of fringe image fB. The phase information is contained in the WT coefficients of the wavelet ridge.

\subsection{Phase Acquirement}

According to the definition in Formula (1), the shift parameter $t$ has a relationship with the phase distribution of the fringe image. We can obtain a conclusion that the phase of the WT coefficient of the optical fringe image is equal to the 
measured object's phase distribution in Formula (10). If the WRT is carried out to the optical fringe image, the position of the wavelet bridge is determined by WT and the maximum module of the WT coefficient. After employing the WRT on the optical fringe image, the wrapped phase map, a phase value that ranges $(-\pi, \pi)$, can be calculated as follows.

$$
\phi(t)=\arctan \left\{\frac{\operatorname{Im}\left[W_{M}\left(p_{r}, t\right)\right]}{\operatorname{Re}\left[W_{M}\left(p_{r}, t\right)\right]}\right\}
$$

Here, Im[] obtains the imaginary of a complex WT coefficient, and Re[] obtains the real parts. If any unwrapping phase method is employed to the wrapped phase map, the phase distribution $\Psi(x)$ of the measured object's surface can be obtained. This acquirement phase method names the phase acquirement method by WRT.

\section{Selection of Scaling Coefficient $p$}

The scaling coefficient $p$ plays an important role in the wavelet ridge extraction algorithm. Its value is very important for the time and accuracy of the obtained phase, so the scaling coefficient vector is usually set by the experiment. During the WRT in actual profilometry, the scaling coefficient vector $p$ is always defined as a set, whose value is an arithmetic sequence with the common difference $\Delta p$.

First of all, the scaling coefficients $\left\{p_{r}\right\}$ that correspond to the wavelet bridge must be in the range of the scaling coefficient vector. We proposed a simple and fast way to configure the vector as follows.

Firstly, suppose that $k_{0}$ is a proportionality ratio of $p$ and $f_{B}$, then Formula (9) can be written as $p=k_{0} / f_{B}$. Moreover, we note that $f_{B} \approx 1 / n$, where $n$ is the pitch pixel number of one fringe. Finally, both range ends of the scaling coefficient vector are evaluated as follows.

$$
\left\{\begin{array}{l}
p_{\text {min }}=\frac{k_{0}}{f_{B_{-} \min }}=k_{0} n_{\text {min }} \\
p_{\text {max }}=\frac{k_{0}}{f_{B_{-} \max }}=k_{0} n_{\text {max }}
\end{array}\right.
$$

where $n_{\min }$ and $n_{\max }$ are respectively where the pixel amount is the narrowest and widest fringe in the fringe image. Both range ends are configured as $p_{\min }$ and $p_{\max }$.

Without a doubt, the common difference $\Delta p$ of the scaling coefficient vector directly influences the accuracy of WRT. The smaller common difference is used in WRT, and the higher accuracy of the phase is obtained. The scaling coefficient vector is discrete, so the location accuracy $p_{r}$ has an uncertainty value of $\Delta p / 2$. This uncertainty value could ignore by using the spatial frequency match filter, which can filter out the first spatial frequency spectrum. The pass-band of the spatial frequency match filer is usually more than $\Delta p / 2$. Finally, through the phase acquirement method by WRT, the wrapped phase can be obtained.

\section{Color Coding Patterns and Absolute Phase Acquirement}

\subsection{White-Light-Interference-Like Color Encoding Fringe Pattern}

A novel color-coding fringe pattern, which is so-called the White-light-interference-like color coding fringe pattern, is employed in this paper. The color coding fringe pattern is shown as Figure 1.

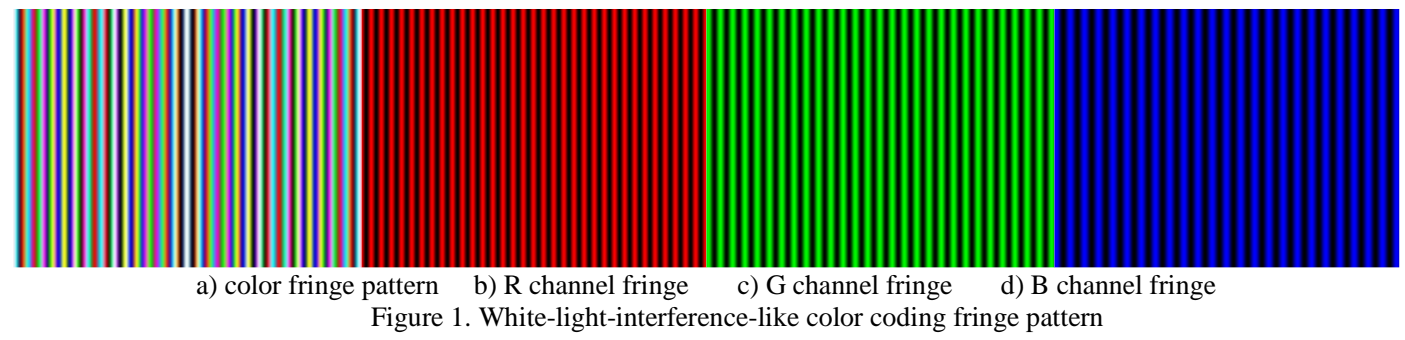

This fringe pattern can be described as follows 


$$
G_{j}(n)=A\left\{1+\cos \left[\frac{2 \pi\left(n-n_{c}\right)}{p i t h_{j}}\right]\right\}
$$

where $\mathrm{Gj}(\mathrm{n})$ is the gray-value in the jth color channel. $\mathrm{j}$ is the $\mathrm{R}, \mathrm{G}, \mathrm{B}$ color channel, $\mathrm{n}$ is the row coordinate of the fringe pattern, nc is the middle of this row, and pithj is the number of coding pixel in the jth channel. In Figure 1, the pitch of the R channel is 30 pixels, and $\mathrm{G}$ and $\mathrm{B}$ have a pitch of 36 and 42 respectively. Because we use the fringe to locate both match pixels between the fringe pattern and fringe image, the column coordinate of fringe pattern can be ignored.

Through a DLP projector, the measured object's surface is projected by only one color fringe pattern. A color camera, which is mounted using the triangle principle and is controlled by one PC, is used to capture the color image. The color image reveals information such as the phase distribution of the measured object's surface (measurement value) and the ambient light (measurement noise). The phase distribution calculated from the captured fringe image (performed as the form of phase change) is related to the 3D coordinate of the measured object.

\subsection{Absolute phase acquirement}

By using WRT, a wrapped phase map can be determined. The wrapped phase in row of the fringe image is shown in Figure 2. In Figure 2, the red line is the wrapped phase acquiring by the $\mathrm{R}$ channel of the fringe image. The pitch in the $\mathrm{R}$ channel is 30 pixels. The green and blue lines, whose pitches are 36 pixels and 42 pixels, are the wrapped phases from the $\mathrm{G}$ and $\mathrm{B}$ channels, respectively. In order to show the detail about the novel color encoding fringe pattern, the dotted rectangle in Figure 2 is enlarged in Figure 3.
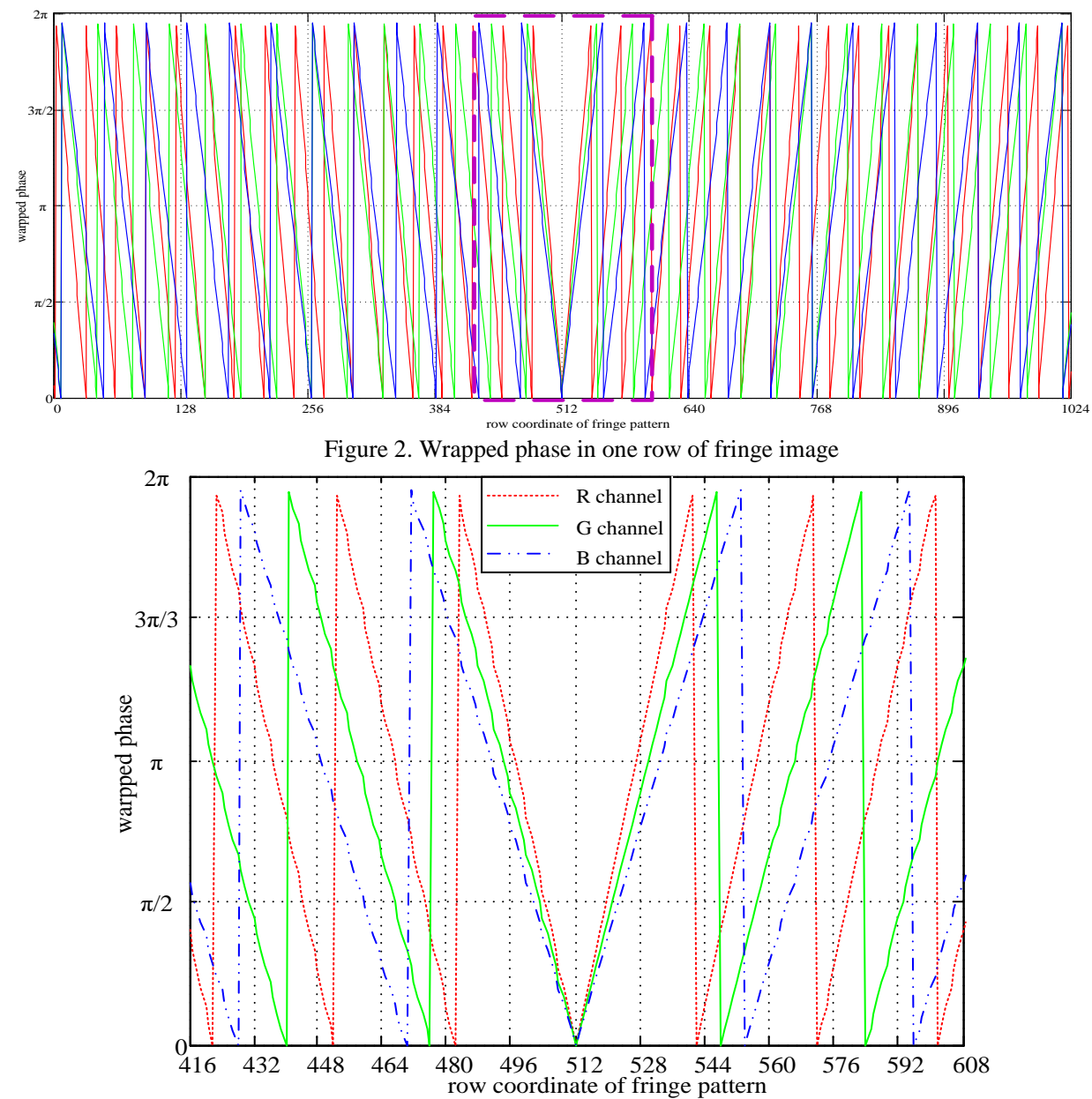

Figure 3. Enlarged scope of purple rectangle in Figure 2

In the color fringe pattern, the unique location feature is the white fringe, which is the coding common origin of the three color channel. It is defined as the phase-crossing of the three-wrapped phase from different color channels. Firstly, we 
must accurately obtain the pixel coordinate of the phase-crossing. There is noise in the fringe image, and so the phasecrossing cannot directly locate the position of the fringe patterns. We define an evaluation function as follows:

$$
E F=\left|\psi_{R}-\psi_{G}\right|+\left|\psi_{G}-\psi_{B}\right|+\left|\psi_{G}-\psi_{R}\right|
$$

where $\psi_{R}, \psi_{G}$ and $\psi_{B}$ are the wrapped phases obtained from the color fringe image using WRT, which is described in section 2. In the phase-crossing, the $E F$ has a minimum value. The optimum phase-crossing can be precisely located in every row of the fringe image using the Levenberg-Marquardt algorithm.

The absolute phase is given as follows

$$
\Psi=2 k_{R} \pi+\psi_{R}=2 k_{G} \pi+\psi_{G}=2 k_{B} \pi+\psi_{B}
$$

where $k_{R}, k_{G}$ and $k_{B}$ are the figure order of R, G and B channel color fringes. Suppose $\Delta k_{R}=\psi_{R} / 2 \pi, \Delta k_{G}=\psi_{G} / 2 \pi$ and $\Delta k_{B}=\psi_{B} / 2 \pi$, and that they are fractional parts. The fractional part corresponds with the wrapped phase in the difference color channel. The key of the absolute phase is obtaining the integer parts $k_{R}, k_{G}$ and $k_{B}$. Suppose that the optimum of phasecrossing is the zeros fringe order and its corresponding phase is 0.

The fringe orders are $k_{B}$ and $k_{G}$ for the two wrapped phases respectively at any point of the object. They are acquired from the $\mathrm{B}$ and $\mathrm{G}$ channels of the color fringe image. The row coordinate of the fringe pattern is $n_{G B}$. The pitch of the superimposed fringe is $n_{G B}$. The following equation can be given:

$$
\left\{\begin{array}{l}
n_{G B}=\left(k_{G}+\Delta k_{G}\right) \text { pitch }_{G}=\left(k_{B}+\Delta k_{B}\right) \text { pitch }_{B} \\
\text { pitch }_{G B}=\frac{\text { pitch }_{G} \times \text { pitch }_{B}}{\text { pitch }_{B}-\text { pitch }_{G}}
\end{array}\right.
$$

From Formula (16), we can obtain:

$$
\begin{gathered}
\left\{\begin{array}{l}
k_{G}=\frac{\text { pitch }_{B}\left(k_{G}-k_{B}+\Delta k_{G}-\Delta k_{B}\right)}{\text { pitch }_{B}-\text { pitch }_{G}} \\
k_{B}=\frac{\text { pitch }_{G}\left(k_{B}-k_{G}+\Delta k_{B}-\Delta k_{G}\right)}{\text { pitch }_{G}-\text { pitch }_{B}}
\end{array}\right. \\
k_{G}-k_{B}=\frac{\text { pitch }_{B}-\text { pitch }_{G}}{\text { pitch }_{G}} k_{B}+\Delta k_{G}-\Delta k_{B}
\end{gathered}
$$

If $k_{G}-k_{B}$ is known, $\Delta k_{G}$ and $\Delta k_{B}$ can be derived from Equation (15). The integer and the fraction of its fringe order are $K_{12}$ and $\Delta K_{12}$. According to the features of the fringe, the following formula is true.

$$
k_{G}-k_{B}= \begin{cases}K_{12} & \psi_{B}-\psi_{G} \leq 0 \\ K_{12}+1 & \psi_{B}-\psi_{G}>0\end{cases}
$$

In the actual measurement, the wrapped phase is having noise, so the formula is rewritten as:

$$
k_{G}-k_{B}=\operatorname{round}\left(\frac{\text { pitch }_{B}-\text { pitch }_{G}}{\text { pitch }_{G}} k_{B}+\Delta k_{G}-\Delta k_{B}\right)
$$

Suppose $k_{G}=k_{R}, k_{B}=K_{12}, \Delta k_{G}=\Delta k_{R}, \Delta k_{B}=\Delta K_{12}$, pitch ${ }_{G}=$ pitch $_{R}$ and pitch ${ }_{B}=$ pitch $_{G B}$. After substituting these into Formula (20), the fringe order corresponding to the absolute phase is acquirement.

Because the zeros fringe order has been located, we can acquire the absolute phase in both sides of the direction of 
pattern pixels. This processing will decrease the time and increase the accuracy of CEFPP by using the smaller pitch of the fringe.

\section{Experiments}

An experiment is carried out to verify the performance of the presented method in this paper. A color-coding cosine fringe pattern is projected onto the measured object, which is a white stand plane, by an Acer H7531D DLP projector. The fringe image is captured by a Hitachi F22 3CCD camera as shown in Figure 4. The baseline between the camera and the projector is approximately $400 \mathrm{~mm}$. The angle between the optical axes of the camera and the projector is about 30 degrees.

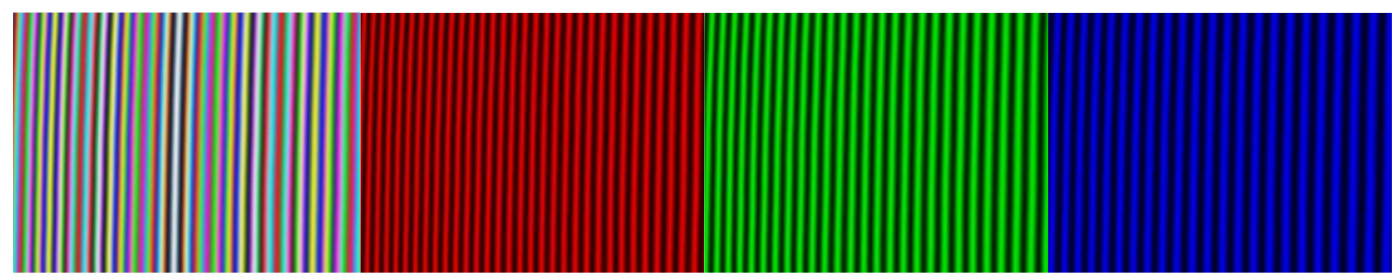

a) Color fringe image b) R channel image c) G channel image d) B channel image

Figure 4. Color fringe image and difference channel image.

The color fringe image is cut to an image resolution of $630 \times 840$ pixels because the image transformation cannot deal with the background. It is easy to detect that the minimum fringe pixel number in different channels, which approximates to $n_{\min }=20$, and the maximum fringe pixels number is $n_{\max }=30$. With the linear calibration method based on the orthogonal White-light-interference-like color coding fringe pattern, the mapping relationship between 3D coordinates and the camera image coordinates is calibrated. The matrix $C$ is the transformation of this relationship and is as follows. The matrix $P$, which is the mapping relationship between projector image coordinates and 3D coordinates, is shown too. The Wrapped phase (WP) of difference color channel is obtained by the wavelet ridge discussed in this paper and is shown in Figure 5.

$$
C=\left(\begin{array}{cccc}
-2.42 & -0.07 & 1.07 & -37.74 \\
0.02 & -2.63 & -0.11 & 54.86 \\
0.00 & 0.00 & 0.00 & 1.00
\end{array}\right) \text { and } P=\left(\begin{array}{cccc}
-2.68 & -0.04 & -0.07 & -125.02 \\
0.05 & -2.73 & -0.55 & 118.16 \\
0.00 & 0.00 & 0.00 & 1.00
\end{array}\right)
$$

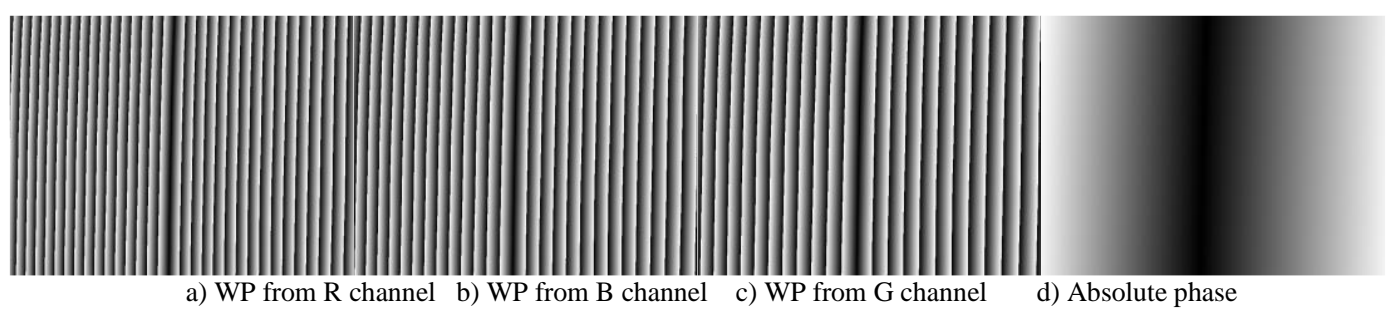

Figure 5. Absolute phase acquired from difference color channel of color fringe image

In Table 1, we provide the mean and standard deviations (SD) for the different position planes within the 3D world coordinate system calibration range.

The profilometry of a complex measured object is carried as a qualitative evaluation of CEFPP presented in this paper. The measured object is shown in Figure 6. The deformed color fringe image is show in Figure 7. The different color channel fringe images shown in Figures $8 \mathrm{a}$ ), b) and c) correspond to the R color channel, the G color channel and the B color channel. Figure $8 \mathrm{~d}$ ) showed the absolute phase measured by the methods presented in Section 4 . The 3D coordinates of the measured object are shown in Figure 9.

Table 1. Measure result of white plane (unit: $\mathrm{mm}$ )

\begin{tabular}{|c|c|c|c|c|c|c|c|}
\hline 3D world coordinate & -150.00 & -100.00 & -50.00 & 0.00 & 50.00 & 100.00 & 150.00 \\
\hline Mean of measure & -151.09 & 100.88 & -50.74 & 0.54 & 50.68 & 100.82 & 151.01 \\
\hline SD of error & 1.42 & 1.24 & 1.07 & 0.70 & 0.98 & 1.15 & 1.37 \\
\hline
\end{tabular}




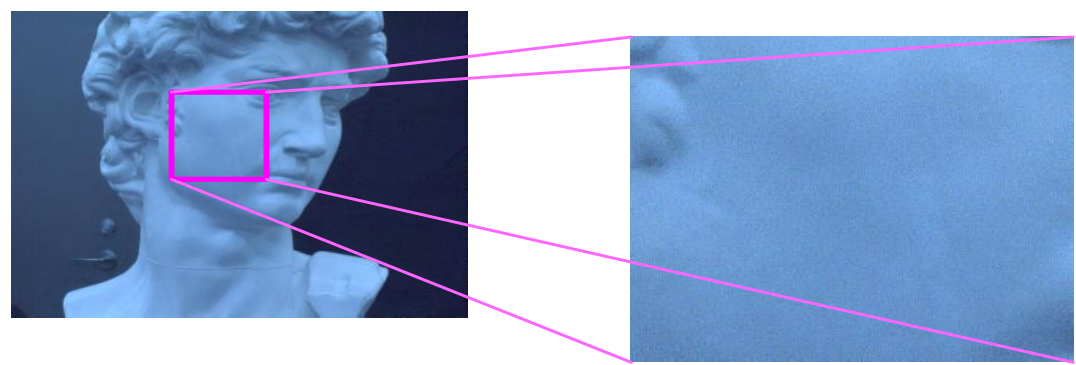

a) Measured object

b) Enlarged scope of purple rectangle of a)

Figure 6. measured object and enlarged scope of purple rectangle of purple rectangle

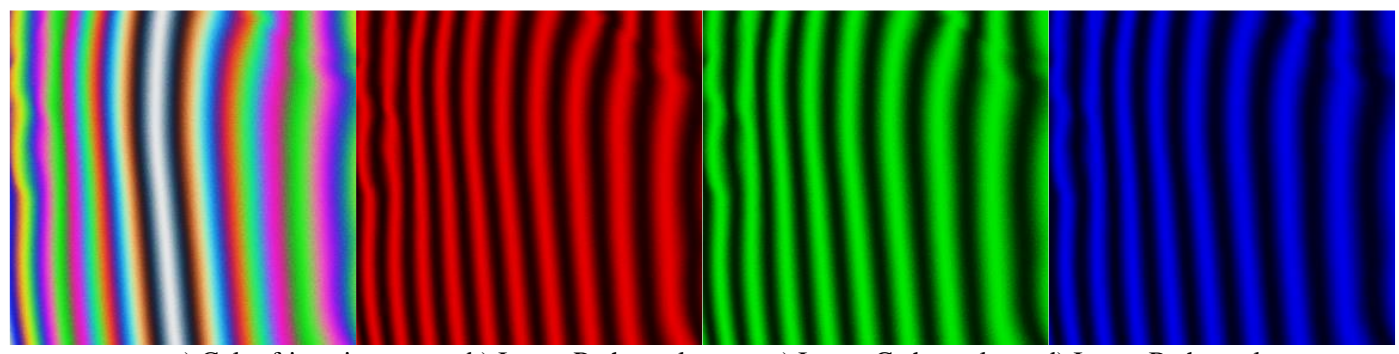

a) Color fringe image b) Image $R$ channel

c) Image $\mathrm{G}$ channel

d) Image B channel

Figure 7. Color fringe image of purple of rectangle in Figure $6 \mathrm{~b}$ )

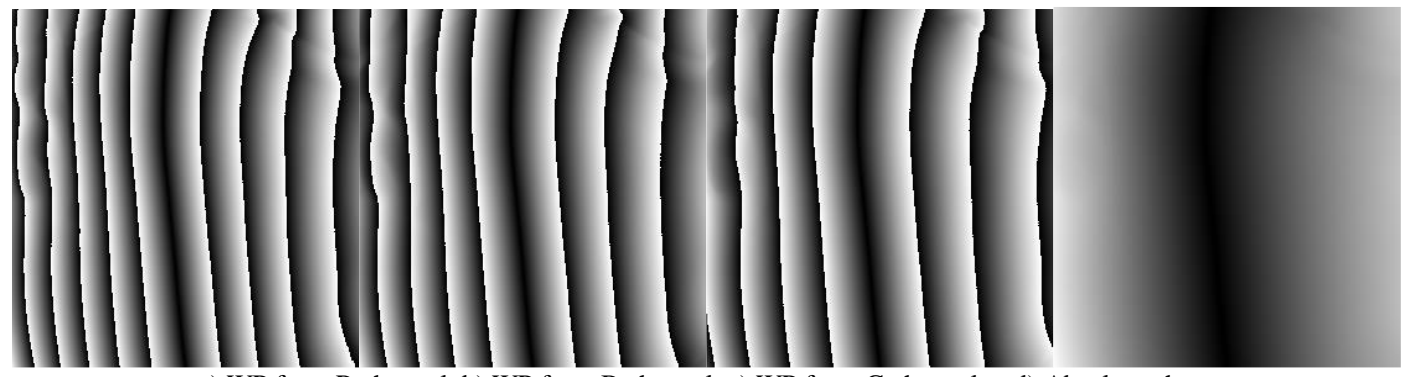

a) WP from R channel b) WP from B channel c) WP from G channel d) Absolute phase Figure 8 . Absolute phase acquired form difference color channel of color fringe image

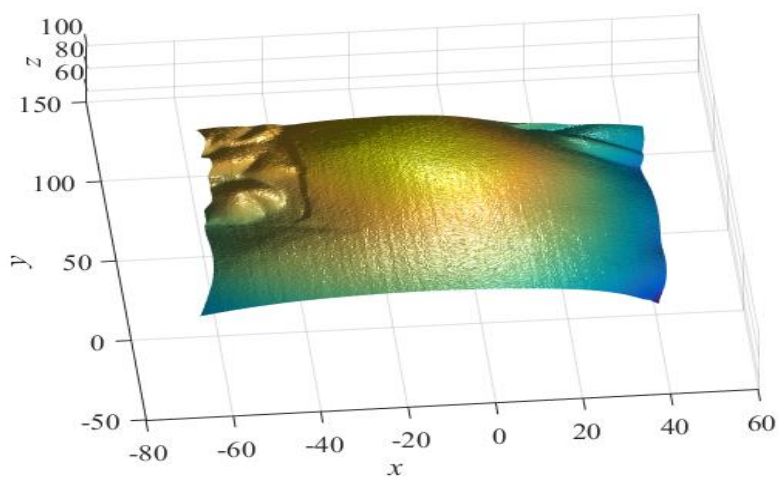

Figure 9. 3D coordinates obtained by our presented method

\section{Conclusions}

A unique color coding profilometry is presented in this paper. It is constituted with three new technologies: the white-lightinterference-like color coding fringe pattern, absolute phase measurement based on WRT and the phase-crossing location. This presented method uses only one color coding pattern to acquire the 3D coordinates of the measured object, which could improve the measurement speed. The phase acquirement technology based on Morlet daughter wavelet ridge definitions in the fringe projection profilometry is theoretically deduced. This derivation reveals that WRT based on Morlet daughter wavelet can be used in color coding fringe projection profilometry. It is clear that the WRT modulus has an inverse relationship with the square root of the wave number of fringe images $F_{\mathrm{b}}$. The reconstruction surface of a complex object is smooth, and it properly displays the face of the measured object. 


\section{Acknowledgements}

This work was partly financially supported through grants from the National Natural Science Foundation of China (No. 60903083 and 61502123), Scientific Planning Issues of Education of Heilongjiang Province (No. GBC1211062), and the Program of New Century Excellent Talents (No. 1155-ncet-008). The authors thank the 3 anonymous reviewers for their helpful suggestions.

\section{References}

1. S.S. Gorthi, P. Rastogi.: "Fringe projection techniques: whither we are?" Optics and lasers in engineering, Vol.48. IMAC-REVIEW-2009-001, pp. 133-140 (2010)

2. Sun, T., Bai, B., Han, C., \& Dong, Z.: “A Color Structured Light Coding and Decoding Method Based on Regional Structured Image with Heterogeneous Applications," International Journal of Signal Processing, Image Processing and Pattern Recognition, Vol.9, No.7, pp.127-136(2016).

3. Su, X. Y., Zhou, W. S., Von Bally, G., \& Vukicevic, D.: "Automated phase-measuring profilometry using defocused projection of a Ronchi grating," Optics Communications, Vol.94, No.6, pp.561-573 (1992).

4. Ling, Y., \& Jiahe, Y.: "The 3D Surface Measurement and Simulation for Turbine Blade Surface Based on Color Encoding Structural Light," International Journal of Signal Processing, Image Processing and Pattern Recognition, Vol.8, No.3, pp.273-280(2015).

5. Quan, C., Tay, C. J., Kang, X., He, X. Y., \& Shang, H. M.: "Shape measurement by use of liquid-crystal display fringe projection with two-step phase shifting," Applied optics, Vol.42, No.13, pp.2329-2335 (2003).

6. Chen, F., Brown, G. M., \& Song, M.: "Overview of three-dimensional shape measurement using optical methods," Optical Engineering, Vol.39, No.1, pp.10-22 (2000).

7. Zhang, S.: "Recent progresses on real-time 3D shape measurement using digital fringe projection techniques." Optics and lasers in engineering, 48(2), 149-158 (2010).

8. Zhang, S., \& Huang, P. S.: "High-resolution, real-time three-dimensional shape measurement," Optical Engineering, Vol.45, No.12, pp.123601-123601 (2006).

9. Jianying, F., Linchao, L., Yang, G., Zeliang, Z., Lei, Y., \& Wei, L.: "Research on color gray code encoding and color components correction in 3d measurement for color object," Vol.6, No.5, pp.271-280(2013).

10. Huang, P. S., Hu, Q., Jin, F., \& Chiang, F. P.: "Color-encoded digital fringe projection technique for high-speed three-dimensional surface contouring," Optical Engineering, Vol.38, No.6, pp.1065-1071 (1999).

11. Pan, B., Kemao, Q., Huang, L., \& Asundi, A.: "Phase error analysis and compensation for nonsinusoidal waveforms in phase-shifting digital fringe projection profilometry," Optics Letters, Vol.34, No.4, pp.416-418 (2009).

12. Xiaoming, S.: "A Study on the Approach of the Structured Light Three-dimensional Measurement. International Journal of u-and e-Service, Science and Technology," Vol.8, No.11, pp.303-316(2015).

13. Hu, Y., Xi, J., Chicharo, J., \& Yang, Z.: "Blind color isolation for color-channel-based fringe pattern profilometry using digital projection," JOSA A, Vol.24, No.8, pp.2372-2382(2007).

14. Yu, X., Shan, L., Cao, S. \& Wu, H.: "The Review of Structured Light Time Encoding Technologies," Journal of Harbin University of Science and Technology, Vol.15, No.18, pp.98-102 (2010).

15. Ma, M., Cao, Y., He, D., Chen, C., \& Wan, Y.: "Grayscale imbalance correcting method based on fringe normalization in RGB tricolor real-time three-dimensional measurement," Optical Engineering, Vol.55, No.3, pp.034102-034102 (2016).

16. Zhang, C., Huang, P. S., \& Chiang, F. P.: "Microscopic phase-shifting profilometry based on digital micromirror device technology," Applied optics, Vol.41, No.28, pp.5896-5904 (2002).

17. Cai, Z., Liu, X., Jiang, H., He, D., Peng, X., Huang, S., \& Zhang, Z.: "Flexible phase error compensation based on Hilbert transform in phase shifting profilometry," Optics Express, Vol.23, No.19, pp.25171-25181 (2015).

18. Liu, C. Y., \& Yen, T. P.: "Digital multi-step phase-shifting profilometry for three-dimensional ballscrew surface imaging," Optics \& Laser Technology, Vol.79, pp.115-123 (2016).

19. Takeda, M., \& Mutoh, K.: "Fourier transform profilometry for the automatic measurement of 3-D object shapes," Applied optics, Vol.22, No.24, pp.3977-3982 (1983).

20. Servin, M., Padilla, M., \& Garnica, G.: "360-degrees profilometry using strip-light projection coupled to Fourier phase-demodulation," Optics express, Vol.24, No. 1, pp.168-179 (2016).

21. Zhong, J., \& Weng, J.: "Dilating Gabor transform for the fringe analysis of 3-D shape measurement," Optical Engineering, Vol.43, No. 4, pp.895-899 (2004).

22. Mallat, S., \& Hwang, W. L.: "Singularity detection and processing with wavelets," IEEE transactions on information theory, Vol.38, No. 2, pp.617-643(1992). 
23. Xu, W., \& Lee, E. J.: "Face Recognition Using Wavelets Transform and 2D PCA by SVM Classifier," International Journal of Multimedia and Ubiquitous Engineering, Vol.9, No. 3, pp.281-290(2014).

24. Telfer, B. A., \& Szu, H. H.: "New wavelet transform normalization to remove frequency bias," Optical Engineering, Vol.31, No. 9, pp.1830-1834(1992).

25. Zhong, J., \& Weng, J.: "Phase retrieval of optical fringe patterns from the ridge of a wavelet transform," Optics letters, Vol.30, No. 19, pp. 2560-2562(2005).

26. Gdeisat, M. A., Burton, D. R., \& Lalor, M. J.: "Spatial carrier fringe pattern demodulation by use of a twodimensional continuous wavelet transform," Applied optics, Vol.45, No. 34, pp.8722-8732(2006).

27. Carmona, R. A., Hwang, W. L., \& Torrésani, B.: "Characterization of signals by the ridges of their wavelet transforms," IEEE transactions on signal processing, Vol.45, No. 10, pp.2586-2590(1997).

28. Watkins, L. R., Tan, S. M., \& Barnes, T. H. "Determination of interferometer phase distributions by use of wavelets," Optics Letters, Vol.24, No. 13, pp.905-907(1999).

29. Afifi, M., Fassi-Fihri, A., Marjane, M., Nassim, K., Sidki, M., \& Rachafi, S.: "Paul wavelet-based algorithm for optical phase distribution evaluation," Optics communications, Vol.211, No. 1, pp.47-51(2002).

30. Cusack, R., Huntley, J. M., \& Goldrein, H. T.: "Improved noise-immune phase-unwrapping algorithm," Applied Optics, Vol.34, No. 5, pp.781-789(1995).

31. Su, X., \& Chen, W.: "Reliability-guided phase unwrapping algorithm: a review," Optics and Lasers in Engineering, Vol.42, No. 3, pp.245-261(2004).

32. Baldi, A.: "Phase unwrapping by region growing," Applied optics, Vol.42, No. 14, pp.2498-2505 (2003).

33. Zhao, H., Chen, W., \& Tan, Y.: "Phase-unwrapping algorithm for the measurement of three-dimensional object shapes," Applied optics, Vol.33, No. 20, pp.4497-4500 (1994).

34. Catherine E. Towers, David P. Towers, and Julian D. C. Jones.: "Optimum frequency selection in multifrequency interferometry," Optics Letters, Vol.28, No. 11, pp.887-889 (2003)

35. Pawłowski, M. E., Sakano, Y., Miyamoto, Y., \& Takeda, M.: "Phase-crossing algorithm for white-light fringes analysis," Optics communications, Vol.260, No. 1, pp.68-72(2006).

36. Lin Y, Zhu X, Zheng Z, et al. "The individual identification method of wireless device based on dimensionality red uction and machine learning”. Journal of Supercomputing, No.5, pp.1-18(2017).

37. Yun Lin, Chao Wang, Zheng Dou. “A Novel Dynamic Spectrum Access Framework Based on Reinforcement Learning for Cognitive Rad io Sensor Networks". Sensors, Vol.16, No.10, pp. 1-22(2016).

38. Lin Y, Wang C, Ma C, et al: "A new combination method for multisensor conflict information," Journal of Supercomputing, Vol.72, No.7, pp. 2874-2890 (2016)

39. Wu Q, Li Y, Lin Y: "The application of nonlocal total variation in image denoising for mobile transmission," Multimedia Tools \& Applications, Vol.76, No.16, pp. 1-13 (2016)

40. Shi C, Dou Z, Lin Y, et al. "Dynamic threshold-setting for RF-powered cognitive radio networks in non-Gaussian noise". Physical Communication, Vol.27, No.4, pp.99-105(2018) 